\title{
Study of the relationship between the conductive system of the internodes in spring bread wheat with lodging resistance and yield traits
}

\author{
Ageeva E.V. ${ }^{*}{ }^{\text {, Leonova I.N. }}{ }^{2}$, Salina E.A. ${ }^{2}$ \\ ${ }^{1}$ Siberian Research Institute of Plant Production and Breeding - Branch of the Institute of Cytology and \\ Genetics, SB RAS, Novosibirsk, Russia \\ ${ }^{2}$ Institute of Cytology and Genetics, SB RAS, Novosibirsk, Russia \\ *email: elenakolomeec@mail.ru
}

Lodging is one of the main problem of reduced productivity of spring bread wheat which can lead to the grain germination and reduced grain quality. Quantitative parameters of the conducting system of the under-ear and second-from-top internodes (the number and diameter of vascular bundles of the parenchymal layer of the stem, the diameter of the internode, the thickness of the mechanical layer thickness and the thickness of the straw) were determined in 11 varieties of spring bread wheat. For this purpose the method of assessing the level of development of the conducting system of the ear internode we used.

The correlations of anatomical and morphological characteristics with the yield, grain quality and resistance to lodging has been found. In the studied genotypes yield varied from 3.51 (Bel) to 5.83 t/ha (Obskaya 2). The most productive varieties originated from mid-ripening (Novosibirskaya 18 and Obskaya 2) and mid-late ripening groups (Triso). The yield of early-maturing varieties was estimated at the level of the average value, which indicates their high potential in the prevailing environments. Three varieties (Velut, Bel and Obskaya 2) had an excess in number of vascular bundles of the parenchymal layer. The Obskaya 2, Velut and Novosibirskaya 18 prevailed over the other wheat varieties in the mechanical layer thickness. It was shown that diameter of the under-ear internode was more than $2 \mathrm{~mm}$. The largest diameter EN1 was recorded in varieties Novosibirskaya $16(3.02 \mathrm{~mm})$, Velut $(2.74 \mathrm{~mm})$ and Novosibirskaya 31 $(2.91 \mathrm{~mm})$. EN2 diameter varied from 2.45 to $4.07 \mathrm{~mm}$. Novosibirskaya 16 was characterized as the thickest among the studied varieties; the diameter of EN2 was $4.07 \mathrm{~mm}$. In general, most of the varieties had good indicators of gluten and protein content. The highest gluten content were shown for the early ripening varieties Novosibirskaya 15 and Novosibirskaya 16 (32.4 and 33.4\% respectively). Analysis of the relationship between anatomical and morphological traits, yield, and grain protein content revealed parameters that correlate with lodging resistance (thickness of the straw and mechanical layer thickness). Obtained results also point to correlation between number of conductive bundles and mechanical layer thickness and the traits that can cause high ear productivity.

Acknowledgments: The anatomical study of the wheat stem was carried out with the support of the budget project of the Institute of Cytology and Genetics, SB RAS No. 0259-2021-0018. The study of protein and gluten content was carried out with the financial support of the Russian Science Foundation grant No. 21-76-30003. 